\title{
Urban Heat Stress Survivability Simulation under Climate Change Scenarios
}

\author{
Norhan Bayomi ${ }^{1}$, Tarek Rakha ${ }^{2}$, John E. Fernandez ${ }^{1}$ \\ ${ }^{1}$ Massachusetts Institute of Technology, Cambridge - MA, USA \\ ${ }^{2}$ Georgia Institute of Technology, Atlanta-Georgia, USA
}

\begin{abstract}
According to IPCC (2014), the global mean temperature is expected to increase from $1.4^{\circ} \mathrm{C}$ to $5.8^{\circ} \mathrm{C}$ by 2100 . The implications will be particularly significant in urban areas as indoor and outdoor comfort levels will be disrupted, leading to significant health impacts. One of the expected impacts is indoor overheating as it has been identified as one of the major causes of thermal discomfort and directly linked to the potential increase in mortality levels in the future. This paper focuses on the potential implications from increased overheating hours on human health in an old low-income residential neighbourhood in Cairo, Egypt. Results from the simulation indicate that under climate change projections, there is a projected increase up to $18 \%$ in indoor overheating with higher health risks for elderly residents.
\end{abstract}

\section{Introduction}

With growing evidence of climate change impacts, rising global temperature is now recognized as one of the major issues facing humans in this century. According to a recent study by Mora et al. (2017), exposure to excess heat is currently one of the inevitable threats to human life. It has been identified that exposure to heat for several consecutive days can lead to deadly health risks, including heat stroke and other heat-related illnesses (Glazer, 2004). Given such risks, there is a rising need for adapting cities to predicted heat-related risks. Such risks will result not only from rising temperatures outdoors but also from buildings' failure to adapt to changing external conditions indoors (Coley \& Kershaw, 2010).

It has been identified that heatwaves are the cause of one of the highest death tolls compared to other weatherrelated hazards (Luber \& McGeehin, 2008) causing increased mortality risks especially for vulnerable populations like the elderly and infants (Kenny, Yardley, Brown, Sigal, \& Jay, 2010). Over the past three decades, a vast body of literature has established a strong relationship between mortality rates and rising outdoor temperature above comfort threshold (Yu et al., 2011). However, indoor temperature can be a significant factor in this relationship. For instance, as outdoor temperatures rise over consecutive days, indoor temperature can rise aggressively, affecting occupant comfort and potentially resulting in heat stress (Sakka, Santamouris, Livada, Nicol, \& Wilson, 2012).
Currently, around $30 \%$ of the global population experience not less than 20 days of excessive heat annually that are considered threatening to human life and is projected that by 2100 three out of four people will be subject to deadly heat stress (Kenny, Flouris, Yagouti, \& Notley, 2018; Mora et al., 2017). Evidence from the 2003 European heatwave underline the risks associated with prolonged exposure to excess heat fatalities that can occur indoors (Valleron \& Boumendil, 2004). At least 35,000 people died as a result of 2003 heatwave with 14,802 deaths were recorded in France alone (United Nation Environment Programme (UNEP), 2004). In 2015, around 110 people died in Egypt from rising temperature, while 580 people were hospitalized due to heat exhaustion after temperature reached $47^{\circ} \mathrm{C}$ (AlAhram, 2015; BBC, 2015).

While there is an extensive body of literature on the effect of extreme outdoor air temperature on mortality, there is a significant limitation of our understanding of the implications of excessive indoor conditions and human health. Furthermore, it is estimated that heat-vulnerable populations like the elderly, individuals with chronic history and infants spend around $80 \%$ to $90 \%$ of their time indoors (Klepeis et al., 2001; Leech, Nelson, Burnett, Aaron, \& Raizenne, 2002). This implies the need to establish a threshold for indoor temperature above which heat stress can occur at a dangerous level to human life.

Overheating risks are expected to increase as a result of climate change with more significant impacts on indoor thermal conditions. Therefore, it is essential to understand the effect of increased temperature in the future on heat stress risks indoor and related health concerns specifically for a vulnerable population. This paper proposes a methodology to evaluate indoor heatrelated risks through the examination of overheating with exposure. The framework of the analysis aims at a) quantifying impacts of future temperature increase on indoor overheating risks and b) classification of building types according to overheating risks to excessive heat. The methodology is applied to an old residential neighbourhood in Cairo, Egypt, with more than $84 \%$ of its population not having access to air conditioning systems (Abouelmagd, 2018; Aga Khan Trust for Culture (AKTC), 2005).

There is extensive literature on the impact of outdoor heat exposure and human health, yet our understanding of indoor heat exposure and health risks under future climate 
projection is still limited (Kenny et al., 2018; Seth H. Holmes, 2016). Thus, the goal of this paper is to highlight the importance of examining this relationship specifically for a vulnerable urban context and implications from climate change projections. The paper is divided into three main sections. The first section presents an overview of overheating risks indoors and implications of excessive heat on human health. The second part of the paper describes the study area, building archetypes, analysis methods, and climate scenarios examined. Finally, section three presents the results of the analysis and outline adaptation strategies.

\section{Extreme Heat and Human Health}

Heatwaves and extreme heat conditions are now considered among the significant threats to human life. Exposure to extreme heat situations often results in heat stress, which is also associated with overheating. Heat stress is a physiological state when the human body is subjected to thermal conditions that are well beyond thermal comfort, and consequently affect basic body functions (Seth H. Holmes, 2016). Heat stress is strongly dependent on the thermoregulation of the human body and thermal comfort resulting from surrounding climatic conditions. Thermal comfort is a function of six main factors; two main personal factors: metabolic rate and clothing level, and four main environmental factors: air temperature, humidity, radiant temperature and airspeed (Parsons \& Kenneth, 2011). The steady range in which the human body maintains constant core temperature is identified as homeothermy zone, as illustrated in Figure 1 below. This range includes the zone of thermal comfort and threshold range of more extreme hot and cold conditions (N. Lacetera, U. Bernabucci, H.H. Khalifa, 2003).

The human body's steady thermal state is within a core temperature of $37^{\circ} \mathrm{C}$ (Ramsey, 1995; Ramsey \& Chai, 1983), and above this range, risk levels start to exacerbate. On the other hand, heat stress occurs when the body fails to maintain heat balance. The three risk ranges presented in Figure 1 above show that by reaching core temperature of $38^{\circ} \mathrm{C}$, the capacity of physical activity starts to decrease, at $39^{\circ} \mathrm{C}$, potentials of heat stroke and heat exhaustion are expected and when the core temperature reached $40^{\circ} \mathrm{C}$, it is considered a life-threatening stage (Barreca, 2012).

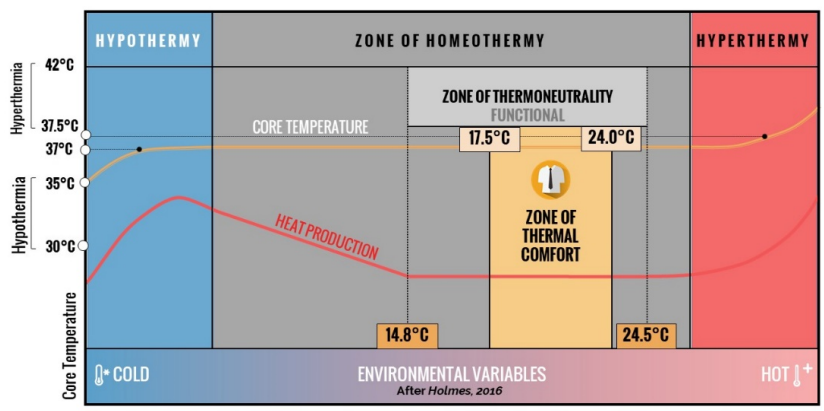

Figure 1: Homeothermy (adapted from (N. Lacetera, U. Bernabucci, H.H. Khalifa, 2003; Seth H. Holmes, 2016))
Individual thermal comfort is directly linked to surrounding climatic conditions. Over the past decade, several studies have investigated the factors governing the relationship between thermal comfort and indoor and outdoor conditions. Hence, the paper focuses on indoor conditions; this section will present an overview of the literature contribution to examining the factors affecting indoor thermal conditions and occupants' sensation of comfort.

The American Society of Heating, Refrigeration and Conditioning Engineers (ASHRAE) standard 55 (2017) has defined thermal comfort as "The state of mind that expresses satisfaction with the thermal environment" with thermal comfort range is between $23^{\circ} \mathrm{C}$ to $28^{\circ} \mathrm{C}$. Given this global definition of comfort, a growing number of studies have developed various models to understand the range of thermal conditions indoors within which occupants will feel most comfortable (Luo et al., 2016). Results from these studies revealed that building characteristics, including construction materials' thermal capacity, orientation, windows properties, and surrounding urban context, play a crucial role in moderating indoor thermal conditions. However, only fewer studies examined the threshold of indoor thermal conditions above which health risks can happen depending on body physiological response.

Basu and Samet's study (2002) is one of the fewer studies that examined indoor temperature and physiological response for elderly residents. The study analyzed skin temperature and heart rate for 42 elderly adults simultaneously with ambient temperature in Baltimore, Maryland, in the USA, over 48 hours period. Results from the study revealed that there is a positive correlation between indoor ambient temperature and skin temperature. As with every $0.56^{\circ} \mathrm{C}$ increase in ambient temperature, skin temperature increased by $0.08^{\circ} \mathrm{C}$.

During the 2003 European heatwave, Stephan et al. (2005) examined 54 patients in a hospital in Paris. Results from this study showed that an indoor air temperature of $40^{\circ} \mathrm{C}$ resulted in elderly and ICU patients' core temperature reaching $38^{\circ} \mathrm{C}$. These core temperatures did not drop until indoor air temperature dropped to between $25^{\circ} \mathrm{C}$ and $27^{\circ} \mathrm{C}$. Also, the study revealed that an indoor air temperature of $38^{\circ} \mathrm{C}$ led to thermoregulation failure despite active body cooling.

In 2012, Kim et al. (2012) examined the impact of indoor condition for low-income housing in South Korea and concluded that increased air temperature negatively correlated with blood pressure in elderly residents and positively linked to increased body temperature. Also, a recent study by Kenny et al. (2017) showed that elderly adults store 1.8 times more heat than younger counterparts under a temperature of $44^{\circ} \mathrm{C}$. The World Health Organization (WHO) has defined groups of the population that are more vulnerable to high and low temperature than others (Ormandy \& Ezratty, 2012). With such definition, it has recommended an indoor temperature range between $18^{\circ} \mathrm{C}$ to $24^{\circ} \mathrm{C}$ that is associated with minimum health risks (Goromosov \& 
WHO, 1968). However, the extent to which this range applies to the vulnerable population with no access to air conditioning systems remains in question.

\section{Heat Stress Indices and Adaptive Capacity}

Heat stress occurs when the body fails to maintain a balanced core temperature around $37^{\circ} \mathrm{C}$. Heat stress indices provide a powerful tool to assess surrounding environmental conditions to predict the probability of thermal strain of the human body (Parsons \& Kenneth, 2011). Generally, heat stress indices denote the impacts of the six main thermal comfort factors describe above on the thermal strain experienced by the human body. Various research has investigated the definitive value of a heat stress index that best represents the temperature perceived by individuals (Epstein \& Moran, 2006). Parsons et al. (2011) presented a comprehensive assessment of different heat stress indices. The study categorized heat stress indices intro three main classes: Rational (based on calculations that take into account heat balance of human body), Empirical (based on the subjective and objective analysis of human physiological response like heart rate and sweating) and Direct (involves measurements to stimulate response of human body to heat) (Epstein \& Moran, 2006; Parsons \& Kenneth, 2011; Ramsey \& Chai, 1983).

Another critical factor to consider in the interrelationship between surrounding climatic conditions and human physiological sensation of comfort is the individuals' adaptation response to heat, also referred to as "Adaptive Capacity” (Hayden, Brenkert-Smith, \& Wilhelmi, 2011). Adaptive capacity refers to the human ability to lessen the impact of exposure to extreme heat using a range of actions to achieve thermal comfort (Hayden et al., 2017). These actions are classified into three main categories, as follows (Kwok \& Rajkovich, 2010):

Physiological Adaptation: linked to the body's ability to adapt to heat through physiological changes like sweating

Psychological Adaptation: based on the individuals' perception of thermal comfort and type of conditions that offer thermally comfortable environment using building control systems, for example.

Behavioural Adaptation: refers to the individuals' response to adapt to heat through a series of adjustments. These adjustments include personal adjustments like changing clothing level and contextual adjustment like opening windows, using air conditioning and closing window blinds to reduce heat stress.

The review above presented a synopsis of the main factors governing the relationship between climatic conditions and the human response to extreme heat and highlighted the main research gaps. The study concerns addressing the gap between indoor climatic conditions and potential health risks specifically for a vulnerable population with a lack of access to adaptation adjustments and air conditioning. The significance of this study stems from establishing a baseline threshold for indoor conditions above which health risks can occur. This would be useful to identify what types of adaptation strategies needed and where they are most effective.

\section{Methods}

The research aims at the identification of risk thresholds associated with increased overheating under future climate projection. In order to provide an understanding of potential overheating risks, a representative urban area is simulated under current and future climate scenarios. The foundation of this analysis is developed through four main sections (Figure 2) through the following steps:

a) Classification of different building types based on construction methods and envelope thermal properties. This is achieved through a series of site surveys that were carried out between July and August 2018 alongside an analysis of findings from The Aga Khan Trust for Culture rehabilitation project (Aga Khan Trust for Culture (AKTC), 2005, 2013).

b) Identification of current users' adaptation strategies in case of extreme heat exposure. This is addressed by conducting 60 personal interviews on types of common adaptation measures, typical clothing levels, ventilation times, equipment types, and types of environmental control systems.

c) Quantifying the impact of increased air temperature on overheating hours indoors. A historical building of three floors that represents $31 \%$ of the existing building stock is modelled in rhinoceros and simulated for annual overheating simulation in grasshopper. Overheating threshold is considered as the operative temperature above ASHRAE comfort range of $26.9^{\circ} \mathrm{C}$ (American Society of Heating \& Conditioning Engineers (ASHRAE), 2017). All buildings were examined with the dependence solely on natural ventilation with no access to any mechanical ventilation system. Among the studied archetypes, a representative case of historic buildings is extensively discussed in this paper.

d) Quantifying overheating impacts across different archetypes. A simulation model of an urban block of 25 buildings that represent the majority of building stock is developed in Urban Modelling Interface (UMI). UMI is an urban performance simulation model developed by the Sustainable Design Lab at Massachusetts Institute of Technology (Reinhart, Dogan, Jakubiec, Rakha, \& Sang, 2013). UMI offers an integrated urban simulation capability including operational energy use, overheating, daylighting, embodied carbon emission and walkability over neighbourhood scale. The simulation module integrates EnergyPlus engine and Radiance. A representative case of each archetype was simulated for hourly indoor operative temperature from January $1^{\text {st }}$ to December $31^{\text {st }}$.

e) Simulations are then carried out for two future climate scenarios: 2020 and 2050 IPCC A2 scenarios (IPCC, 2014). Climate Change Weather generator tool developed by the sustainable energy research group in Southampton University (Jentsch, James, Bourikas, \& Bahaj, 2013) is used for morphing 2020 and 2050 weather files under A2 scenario.

f) Analyzing the effect of prolonged heat exposure on health risks. A hypothetical model of a 65 years old 
male resident is simulated using Ladybug human comfort simulation module to measure potential health implications from increased indoor temperature (Mostapha Sadeghipour \& Michelle, 2013).

To assess potential health impacts, Heart Rate (HR) is used as the primary physiological indicator for potential risk (Zamanian et al., 2017). There are several other indices to reflect physiological response under heat exposure, such as skin temperature, core temperature, sweat rate, and dehydration risks. For this study, heart rate is used as the main analysis metric. Heart Rate (HR) is a metric developed by Fuller and Brouha (1966) to attest thermal stress on the human body. A hypothetical human male model is developed in grasshopper and honeybee with a representative body characteristic for a 65 years old Egyptian male who is acclimatized to heat. The model is examined for a sedentary activity level in the main bedroom space on each floor of the examined archetype. Simulations were run for current conditions, 2020 and 2050 climate scenarios for the extreme hot week from August $19^{\text {th }}$ to August $25^{\text {th }}$.

This will assist in quantifying the threshold of indoor conditions that can potentially cause health risks. The rationale behind this simulation is to test impacts on the elderly who are considered most vulnerable to heat exposure, as compared to young adults. Although infants are also prone to heat-related risks, they are not included in this analysis.

Simulation Assumptions: occupancy and lighting schedules were based on the personal interviews carried out during site surveys. Equipment loads and lighting density were assumed based on a local study (Attia, Evrard, \& Gratia, 2012) that developed a representative energy simulation database for building stocks in Egypt. Infiltration rates used were equal to 0.3 air changes per hour $(\mathrm{ACH})$, assuming that the majority of buildings have relatively poor airtightness. All buildings' windows were assumed single pane clear glass without coating.

\section{Characteristics of the Study Area}

Al-Darb AlAhmar is a low-income neighbourhood in Cairo, Egypt. The area has a mix of old historical buildings from the $1700 \mathrm{~s}$, buildings that were built during the late $20^{\text {th }}$ century and buildings that were built in the period between 2010 and 2014. The total studied area is 2.8 acres, and it was specifically selected for its unique architectural character and diversity of building types and economic challenges. Living standards are primarily considered for low-income, where air conditioning units are not common and rarely installed. Ceiling fans and portable fans are the most common apparatus used for ventilation. There are eight main archetypes identified from the site survey, with the characteristics of envelopes' thermal properties vary with construction methods into two main categories. Characteristic of building envelopes in each archetype identified based on site survey and Attia's database for Egypt's construction materials (Attia \& Wanas, 2012).
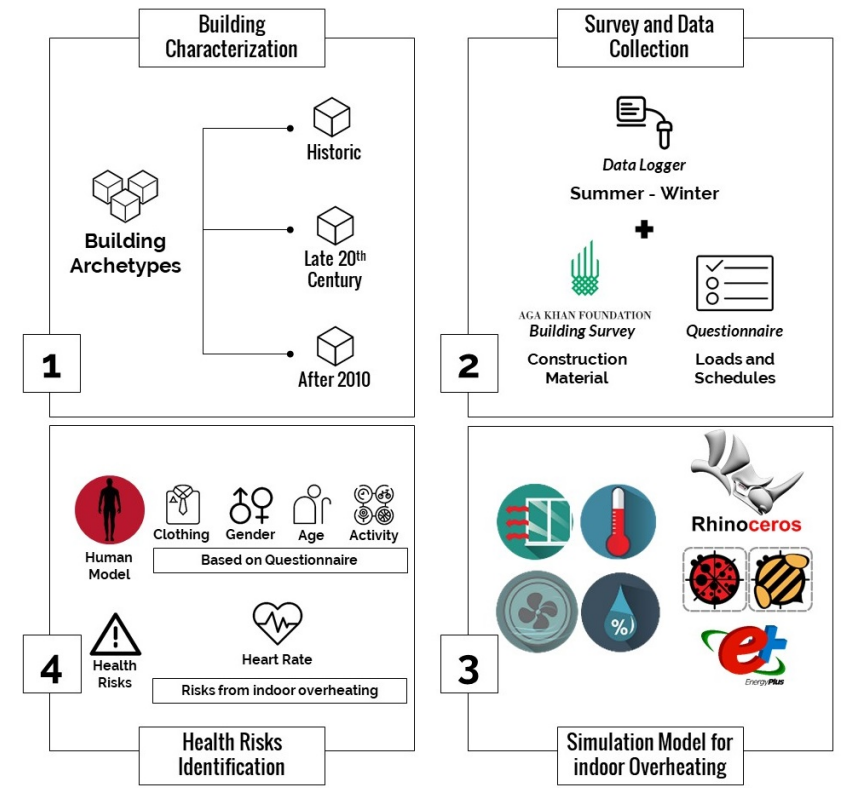

Figure 2: Analysis framework and methods used.

The floor area of the residence examined in the study range between $30 \mathrm{~m}^{2}$ and $100 \mathrm{~m}^{2}$, with an average area of $80 \mathrm{~m}^{2}$. Onset Hobo data loggers were installed in 15 representative archetypes from July $6^{\text {th }}$ to July $12^{\text {th }}$ and from August $8^{\text {th }}$ to August $15^{\text {th }}$. Indoor temperature and relative humidity readings are used to analyze indoor climatic conditions and validate simulation results. The accuracy of the temperature loggers used was $\pm 0.21^{\circ} \mathrm{C}$. All loggers were placed in the zones with sufficient air movement and away from any internal heat sources and solar radiation. Outdoor air temperature was collected from the nearest weather station that is located $4.9 \mathrm{~km}$ away from the analysis area. Outdoor temperature readings indicate high temperature from July $7^{\text {th }}$ until July $12^{\text {th, }} 2018$.

\section{Results}

Figure 3 presents the simulated indoor air temperature and recorded air measurements. Data from buildings' survey and questionnaires were used to calibrate simulation model with actual conditions.

As shown in Figure 3 below, simulation mean square root error decreased substantially after adding questionnaire data. The questionnaire was tailored to address information such as occupancy times, the number of equipment owned, adjustments residents use to regulate temperature and ventilation times. All these data we arranged by archetype and added to the simulation model.

Overheating simulation results indicate that the majority of overheating hours occurs between June and October, with a higher frequency between July and August as presented in Figure 4-a below. The daily distribution shows that during summer months from May to September, most of the overheating hours occur in the nighttime from $6 \mathrm{pm}$ to 6 am (Figure 4-b). The large concentration of overheating hours in nighttime poses threats related to potential disruptive sleep from increased temperature. 


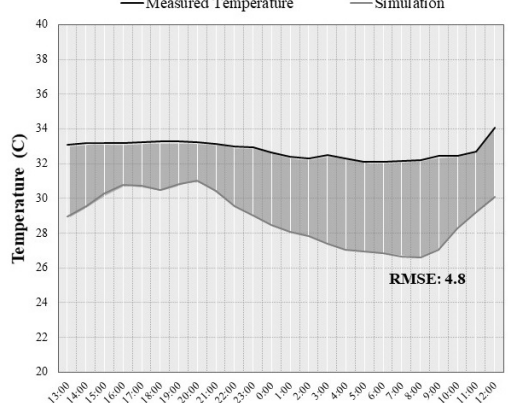

a. Initial Simulation results before integrating data on occupancy schedules and lighting and equipment loads

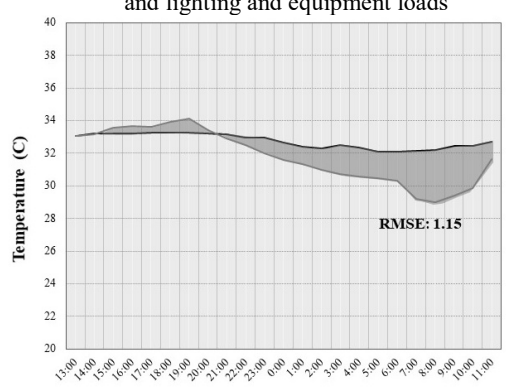

b. Simulation results after calibration with site data and personal occupancy questionnaire

Figure 3: Simulation results for indoor air temperature and recorded summer measurements.

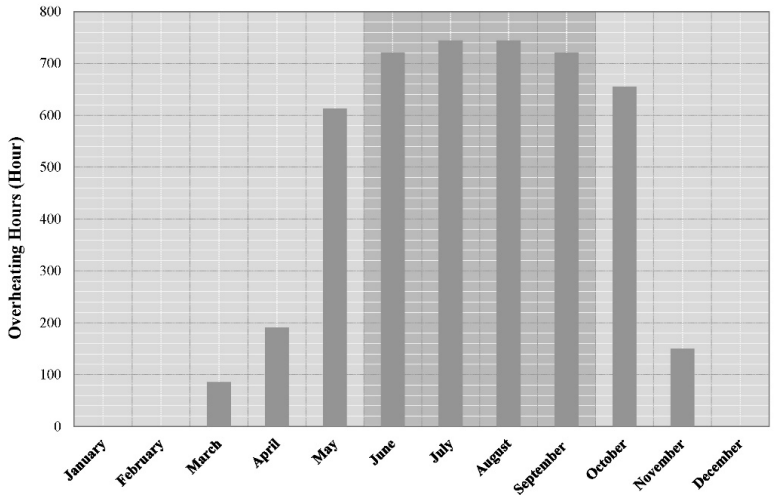

a. Annual overheating simulation and monthly distribution

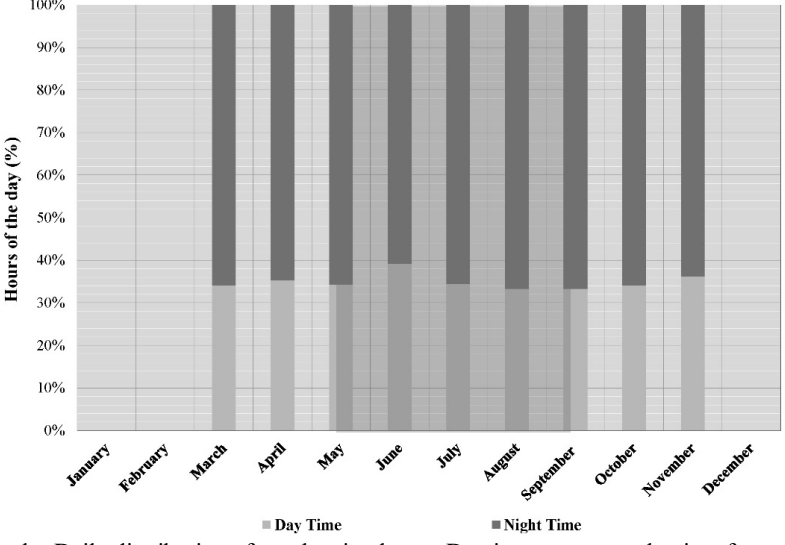

b. Daily distribution of overheating hours. Daytime represents the time from 6 am to $6 \mathrm{pm}$, and Nighttime represents from $6 \mathrm{pm}$ to $6 \mathrm{am}$

Figure 4: Overheating monthly and daily simulation results for a representative historic building.

Operative temperature simulations for typical summer week and extreme hot week shows that higher floors have higher average operative temperature than lower floors. Hourly operative temperature simulation for the month of July revealed that during the first three days of the month,

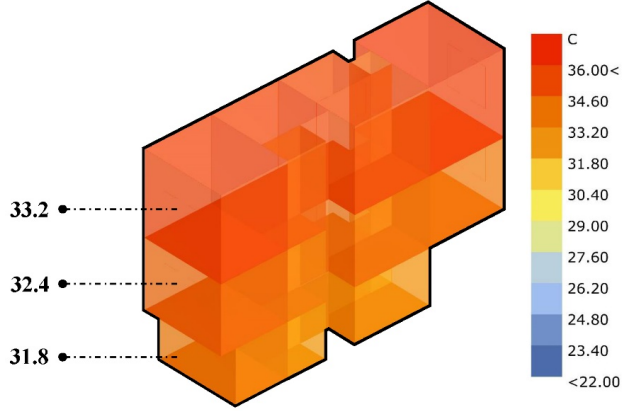

a. Average hourly operative temperature for a typical summer week $\left(15^{\text {th }}\right.$ to

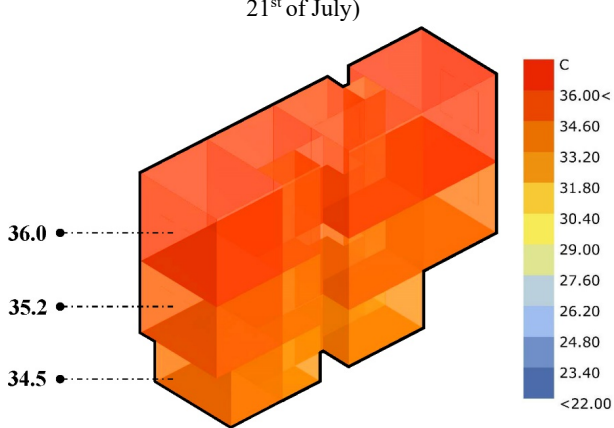

b. Average hourly operative temperature for an extremely hot week ( $19^{\text {th }}$ to $25^{\text {th }}$ of August)

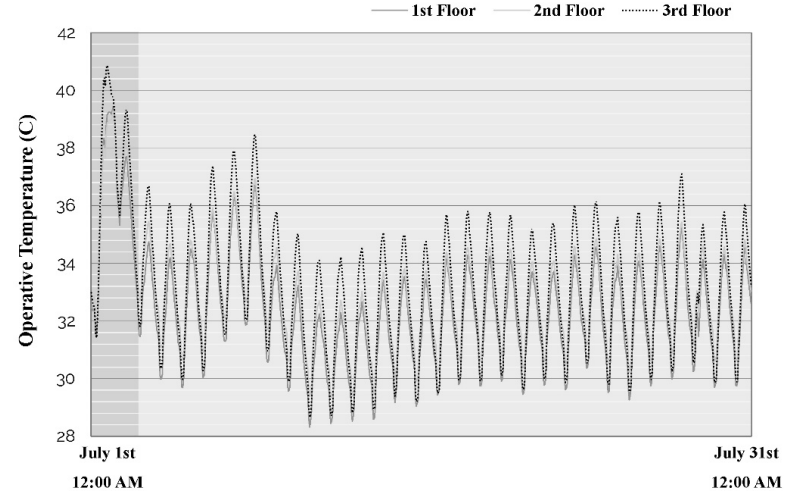

c. Hourly Operative temperature for July. The highlighted region represents the highest observed operative temperature above the monthly average from July $1^{\text {st }}$ to July $3^{\text {rd }}$

Figure 5: a. Average operative for a typical summer week in July, b. Operative temperature for the extreme hot week and c. Hourly simulation for each floor in July. temperature exceeds a monthly average of $32.8^{\circ} \mathrm{C}$, as presented in Figure 5-b.

The subsequent stage was to use validation for individual archetypes to develop an urban template for UMI inputs. An urban area of 1.2 acres is simulated for annual overheating hours above $26.9^{\circ} \mathrm{C}$. Simulation results highlight that older building has relatively lower overheating hours as compared to archetypes after the late $20^{\text {th }}$ century. Upper floor across all archetypes had higher annual overheating hours compared to other floors in the same building.

The average annual overheating in historic buildings ranged from 4800 hours to 6300 hours, as shown in Figure 6 -a. On the other hand, archetypes from the late $20^{\text {th }}$ century and those, which were built after 2010, had average annual overheating hours ranging between 3000 hours to 3500 hours annually as illustrated in Figure 6-b. 


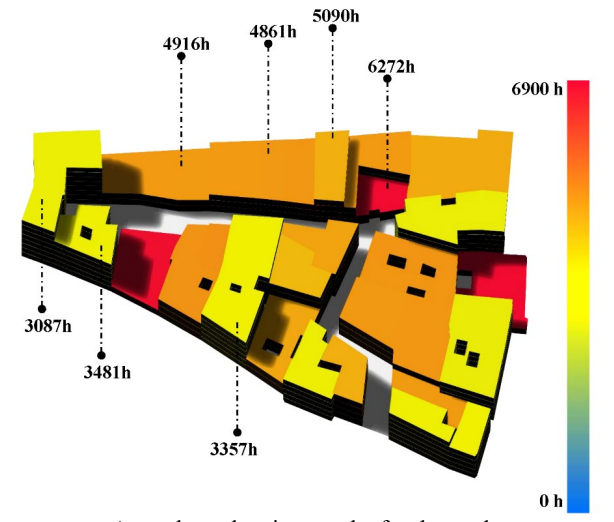

a. Annual overheating results for the study area

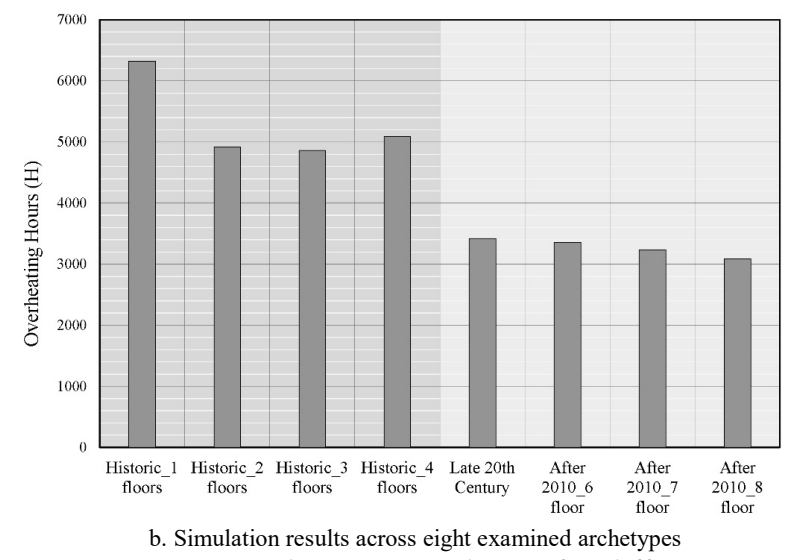

Figure 6: Overheating simulation for different Archetypes.

These results indicate higher overheating in historical archetypes. The following step is the evaluation of the projected increase in overheating hours across different archetypes under 2020 and 2050 A2 climate scenarios.

By running simulation under 2020 and 2050 scenarios, several trends have been identified. Generally, there is a significant increase in overheating hours, especially for archetypes from the late $20^{\text {th }}$ century and archetypes that were built after 2010. Figure 7 below presents overheating increase across all eight archetypes for current conditions, 2020 and 2050 scenarios. Results show that archetypes from the period of the late $20^{\text {th }}$ century and after 2010 have an average increase in overheating by $12 \%$ and $18 \%$ by 2020 and 2050 respectively. Notably, historic buildings had an average increase of 5\% and $6 \%$.

With the understanding of the expected increase in overheating hours across different archetypes, we focus on potential health impacts associated with increased temperature. Results from simulation indicate there is a potential health risk associated with increased overheating in the examined archetype. Figure 8 represents Heart Rate for each floor under the current scenario and 2050 scenario. It can be noted that there is a potential risk from increased heart rate, especially between 15:00 pm to midnight. Also, there is a relationship between heart rate per minute and floor location, which coincides with the aforementioned operative temperature simulations.

These results entail a potential relationship between operative temperature and heart rate per minute.

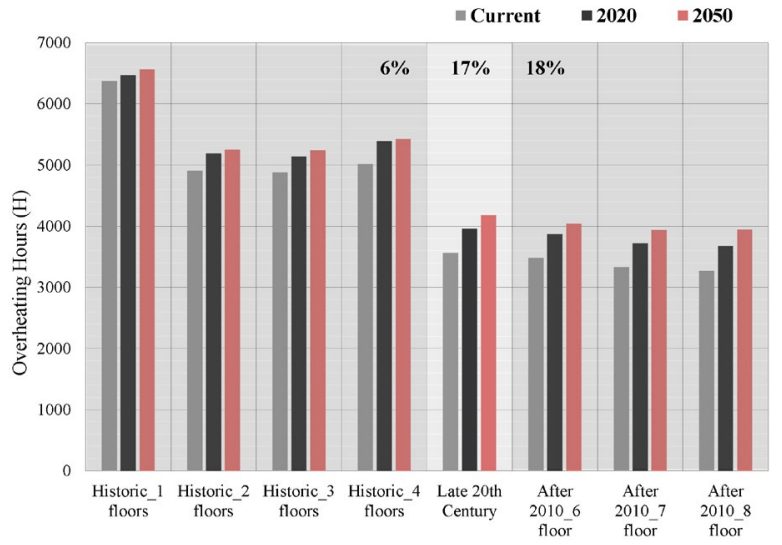

Figure 7: Annual overheating simulation results for current conditions, 2020 and 2050 scenarios.

Regression analysis revealed that there is a positive correlation between operative temperature and heart rate per minute $\left(R^{2}=0.859\right)$. As a result, for three consecutive hours of exposure time to an indoor operative temperature of $34^{\circ} \mathrm{C}$, heart rate exceeds 90 beats per minute. This limit represents a warning zone for health risks, especially for elderly residents with chronic diseases history (Błażejczyk Krzysztof, 2011). Finally, looking at results from the current condition, 2020 and 2050, there is an expected average increase of $3 \%$ in heart rate levels between current and 2050-climate scenario for the examined archetype (Figure 8-b).

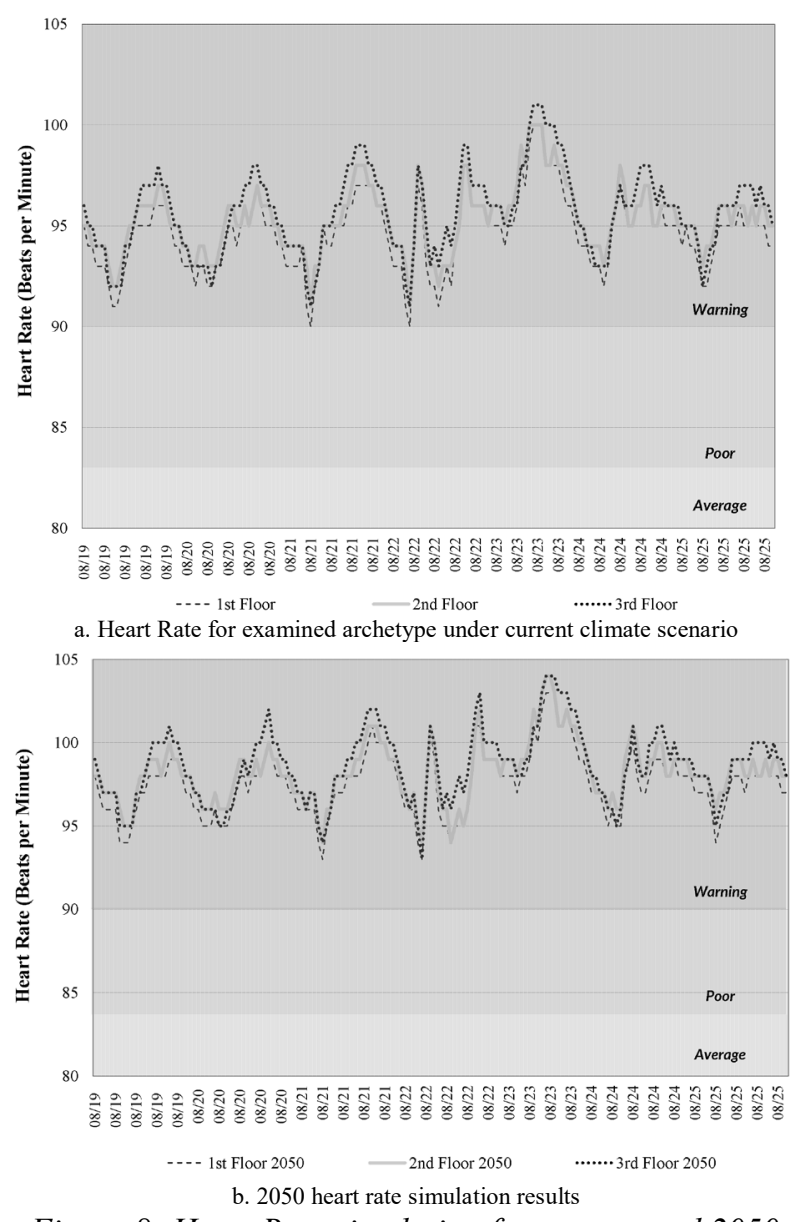

Figure 8: Heart Rate simulation for current and 2050 climate scenario. 


\section{Conclusion}

The study aimed to undertake an in-depth investigation of potential health risks associated with increased indoor overheating for vulnerable populations with limited access to mechanical ventilation. The goal was to investigate the threshold for indoor conditions that could result in health risks specifically for elderly residents. A quantitative analysis using residents' questionnaire has been designed to develop a representative simulation model. Results from the simulation highlighted strong evidence of overheating across examined archetypes. Generally, the upper floors showed a higher number of overheating hours, especially for historical archetypes. This can be explained by the wall bearing structure system that was commonly used in a historic building. For a three storey building, the higher floor had a wall thickness of $12.5 \mathrm{~cm}$ compared to a lower floor of $40 \mathrm{~cm}$ thickness.

The second stage of the analysis focused on developing an urban simulation model to investigate the potential increase in overheating over a large urban scale. This analysis was carried out in UMI using real occupancy scenario from the residents' questionnaire. Three simulation scenarios were selected and examined to explore the potential increase in overheating.

Results from the simulation revealed that there is a significant increase in overheating across all archetypes, especially in historic buildings. This can be directly linked to a small floor area with high population density per $\mathrm{m}^{2}$ compared to newer buildings. As historic archetypes were initially designed to accommodate one family and now a change of use resulted in higher population density compared to newer archetypes. On the other hand, results from the 2020 and 2050 climate scenario revealed a faster increase in archetypes from the late $20^{\text {th }}$ century and late 2010.

Health implication associated with increased overheating showed that for historic buildings, there is a threshold of exposure under an operative temperature of $34^{\circ} \mathrm{C}$ for more than three consecutive hours. The main limitation of the analysis is focusing on a single building type with the assumption of consistent occupancy and ventilation scenarios. Further research would investigate impacts of changing ventilation and occupancy schedules with other passive strategies and testing these parameters for other thermal comfort indices such as Wet Bulb Global Temperature (WBGT), Predicted Heat Strain (PHS) and Sweat Rate (SR) across varying activity levels and occupants' types.

\section{References}

Abouelmagd, D. (2018). Assessing the Aga Khan Conservation and Urban Revitalization Projects (The Case Studies of Al-Darb Al-Ahmar in Cairo, and the Humayun's Tomb - Nizamuddin Basti in Delhi). SSRN Electronic Journal, 1-12.

Aga Khan Trust for Culture (AKTC). (2005). Cairo : Urban Regeneration in the Darb al-Ahmar District.

Aga Khan Trust for Culture (AKTC). (2013). Urban Conservation and Regeneration of Heritage Areas in
Egypt Aga Khan Darb Al-Ahmar Project Model. Geneva, Switzerland.

AlAhram. (2015, August 20). Egypt's Heatwave Death Toll reaches 110.pdf. Al-Ahram Online.

American Society of Heating, R., and A.-, \& Conditioning Engineers (ASHRAE). (2017). Standard 55- Thermal Comfort.

Attia, S., Evrard, A., \& Gratia, E. (2012). Development of benchmark models for the Egyptian residential buildings sector. Applied Energy, 94(2012), 270-284.

Attia, S., \& Wanas, O. (2012). The Database of Egyptian Building Envelopes (Debe): a Database for Building Energy Simulations. In Fifth National Conference of IBPSA-USA (pp. 96-103). Madison, Wisconsin.

Barreca, A. I. (2012). Climate change, humidity, and mortality in the United States. Journal of Environmental Economics and Management, 63(1), 19-34.

Basu, R., \& Samet, J. M. (2002). An exposure assessment study of ambient heat exposure in an elderly population in Baltimore, Maryland. Environmental Health Perspectives, 110 (12), 1219-1224.

BBC. (2015, August 12). Egypt heatwave leaves 61 people dead.pdf. $B B C$ News.

Błażejczyk Krzysztof, K. A. (2011). Bioclimatic conditioning of recreation and tourism in Poland. Warszawa, Poland. Retrieved from http://rcin.org.pl

Coley, D., \& Kershaw, T. (2010). Changes in internal temperatures within the built environment as a response to a changing climate. Building and Environment, 45(1), 89-93.

Edenhofer, O., Pichs-Madruga, R., Sokona, Y., Minx, J. C., Farahani, E., Susanne, K., ... Zwickel, T. (2014). Climate Change 2014: Mitigation of Climate Change. Working Group III Contribution to the Fifth Assessment Report of the Intergovernmental Panel on Climate Change.

Epstein, Y., \& Moran, D. S. (2006). Thermal Comfort and the Heat Stress Indices Heat Balance and Heat Exchange. Industrial Health, 44, 388-398.

Fuller, F. H., \& Brouha, L. (1966). New engineering methods for evaluating the job environment. ASHRAE $J$.

Glazer, J. L. (2004). Management of Heatstroke and Heat Exhaustion - American Family Physician, 71(11), 18. Retrieved from papers3://publication/uuid/0DA40D7F-957B-4735A5E0-E59CDB8B2049

Goromosov, M., \& WHO. (1968). The physiological basis of health standards for dwellings. Retrieved from http://apps.who.int/iris/handle/10665/39749

Hayden, M. H., Brenkert-Smith, H., \& Wilhelmi, O. V. (2011). Differential Adaptive Capacity to Extreme Heat: A Phoenix, Arizona, Case Study. Weather, Climate, and Society, 3(4), 269-280.

Hayden, M. H., Wilhelmi, O. V., Banerjee, D., Greasby, T., Cavanaugh, J. L., Nepal, V., ... Gower, S. (2017). Adaptive Capacity to Extreme Heat: Results from a 
Household Survey in Houston, Texas. Weather, Climate, and Society, WCAS-D-16-0125.1.

IPCC. (2014). Technical Summary- Climate Change: Impacts, Adaptation, and Vulnerability. Climate Change 2014: Impacts, Adaptation, and Vulnerability. Part A: Global and Sectoral Aspects. Contribution of Working Group II to the Fifth Assessment Report of the Intergovernmental Panel on Climate Change. Geneva, Switzerland.

Jentsch, M. F., James, P. A. B., Bourikas, L., \& Bahaj, A. B. S. (2013). Transforming existing weather data for worldwide locations to enable energy and building performance simulation under future climates. Renewable Energy, 55, 514-524.

Kenny, G. P., Flouris, A. D., Yagouti, A., \& Notley, S. R. (2018). Towards establishing evidence-based guidelines on maximum indoor temperatures during hot weather in temperate continental climates. Temperature, 8940(May), 1-26.

Kenny, G. P., Poirier, M. P., Metsios, G. S., Boulay, P., Dervis, S., Friesen, B. J., ... Flouris, A. D. (2017). Hyperthermia and cardiovascular strain during extreme heat exposure in young versus older adults. Temperature, 4(1), 79-88.

Kenny, G. P., Yardley, J., Brown, C., Sigal, R. J., \& Jay, O. (2010). Heat stress in older individuals and patients with common chronic diseases. Cmaj, 182(10), 10531060

Kim, Y.-M., Kim, S., Cheong, H.-K., Ahn, B., \& Choi, K. (2012). Effects of Heat Wave on Body Temperature and Blood Pressure in the Poor and Elderly. Environmental Health and Toxicology, 27, e2012013.

Klepeis, N. E., Nelson, W. C., Ott, W. R., Robinson, J. P., Tsang, A. M., Switzer, P., ... Engelmann, W. H. (2001). The National Human Activity Pattern Survey. Lawrence Berkeley National Laboratory (Vol. 11).

Kwok, A. G., \& Rajkovich, N. B. (2010). Addressing climate change in comfort standards. Building and Environment, 45(1), 18-22.

Leech, J. A., Nelson, W. C., Burnett, R. T., Aaron, S., \& Raizenne, M. E. (2002). It is about time: A comparison of Canadian and American time-activity patterns. Journal of Exposure Analysis and Environmental Epidemiology, 12(6), 427-432.

Luber, G., \& McGeehin, M. (2008). Climate Change and Extreme Heat Events. American Journal of Preventive Medicine, 35(5), 429-435.

Luo, M., de Dear, R., Ji, W., Bin, C., Lin, B., Ouyang, Q., \& Zhu, Y. (2016). The dynamics of thermal comfort expectations: The problem, challenge, and implication. Building and Environment, 95, 322-329.

Mora, C., Dousset, B., Caldwell, I. R., Powell, F. E., Geronimo, R. C., Bielecki, C. R., ... Trauernicht, C. (2017). Global risk of deadly heat. Nature Climate Change, 7(7), 501-506.

Mostapha Sadeghipour, R., \& Michelle, P. (2013). Ladybug: A Parametric Environmental Plugin for Grasshopper to help designers create an environmentally-conscious design. In 13th Conference of International Building Performance Simulation Association (Vol. 13, pp. 3128-3135). Chambery. France.

N. Lacetera, U. Bernabucci, H.H. Khalifa, B. R., and A. N. (2003). Interactions between climate and animal production (EAAP Techn). Wageningen Academic.

Ormandy, D., \& Ezratty, V. (2012). Health and thermal comfort: From WHO guidance to housing strategies. Energy Policy, 49, 116-121.

Parsons \& Kenneth, C. (2011). Assessment of Heat Stress and Heat Stress Indices. In Encyclopedia of Occupational Health and Safety (pp. 1-12). Geneva, Switzerland: International Labor Organization.

Ramsey, J. D. (1995). Task performance in heat: A review. Ergonomics.

Ramsey, J. D., \& Chai, C. P. (1983). Inherent variability in heat-stress decision rules. Ergonomics, 26(5), 495504.

Reinhart, C. F., Dogan, T., Jakubiec, J. A., Rakha, T., \& Sang, A. (2013). UMI - An Urban Simulation Environment for Building Energy Use, Daylighting, and Walkability. In 13th Conference of International Building Performance Simulation Association (pp. 79-82). Chambery. France.

Sakka, A., Santamouris, M., Livada, I., Nicol, F., \& Wilson, M. (2012). On the thermal performance of low income housing during heat waves. Energy and Buildings, 49, 69-77.

Seth H. Holmes, T. P. \& A. W. (2016). Overheating and passive habitability: indoor health and heat indices. Building Research \& Information, 44(1), 1-19.

Stéphan, F., Ghiglione, S., Decailliot, F., Yakhou, L., Duvaldestin, P., \& Legrand, P. (2005). Effect of excessive environmental heat on core temperature in critically ill patients. An observational study during the 2003 European heat wave. British Journal of Anaesthesia, 94(1), 39-45.

United Nation Environment Programme (UNEP). (2004). Impacts of Summer 2003 heat wave in Europe. Environment Alert Bulletin. Nairobi, Kenya.

Valleron, A. J., \& Boumendil, A. (2004). Épidémiologie et canicules: Analyses de la vague de Chaleur 2003 en France. Comptes Rendus - Biologies, 327(12), 11251141.

Yu, W., Mengersen, K., Hu, W., Guo, Y., Pan, X., \& Tong, S. (2011). Assessing the relationship between global warming and mortality: Lag effects of temperature fluctuations by age and mortality categories. Environmental Pollution, 159(7), 17891793.

Zamanian, Z., Sedaghat, Z., Hemehrezaee, M., \& Khajehnasiri, F. (2017). Evaluation of environmental heat stress on physiological parameters. Journal of Environmental Health Science and Engineering, 15(1), 1-8. 